\title{
Reproducibility of adenosine stress cardiovascular magnetic resonance in multi-vessel symptomatic coronary artery disease
}

\author{
Sharon Chih*1,2, Peter S Macdonald1,2,3, Michael P Feneley 1,2,3, Matthew Law33, Robert M Graham 1,2,3 and \\ Jane A McCrohon 2,3
}

\begin{abstract}
Purpose: First-pass perfusion cardiovascular magnetic resonance (CMR) is increasingly being utilized in both clinical practice and research. However, the reproducibility of this technique remains incompletely evaluated, particularly in patients with severe coronary artery disease (CAD). The purpose of this study was to determine the inter-study reproducibility of adenosine stress CMR in patients with symptomatic multi-vessel CAD and those at low risk for CAD.

Methods: Twenty patients (10 with CAD, 10 low risk CAD) underwent two CMR scans $8 \pm 2$ days apart. Basal, mid and apical left ventricular short axis slices were acquired using gadolinium $0.05 \mathrm{mmol} / \mathrm{kg}$ at peak stress (adenosine, $140 \mu /$ $\mathrm{kg} / \mathrm{min}, 4 \mathrm{~min}$ ) and rest. Myocardial perfusion was evaluated qualitatively by assessing the number of ischemic segments, and semi-quantitatively by determining the myocardial perfusion reserve index (MPRi) using a normalized upslope method. Inter-study and observer reproducibility were assessed--the latter being defined by the coefficient of variation (CoV), which was calculated from the standard deviation of the differences of the measurements, divided by the mean. Additionally, the percentage of myocardial segments with perfect agreement and inter- and intra-observer MPRi correlation between studies, were also determined.
\end{abstract}

Results: The CoV for the number of ischemic segments was 31\% with a mean difference of $-0.15 \pm 0.88$ segments and 91\% perfect agreement between studies. MPRi was lower in patients with CAD $(1.13 \pm 0.21)$ compared to those with low risk CAD (1.59 \pm 0.58$), p=0.02$. The reproducibility of MPRi was $19 \%$ with no significant difference between patients with CAD and those with low risk CAD $(p=0.850)$. Observer reproducibility for MPRi was high: inter-observer CoV 9\%, $r=0.93$ and intra-observer CoV 5\%, $r=0.94$. For trials using perfusion CMR as an endpoint, an estimated sample size of 12 subjects would be required to detect a two-segment change in the number of ischemic segments (power 0.9, a 0.05).

Conclusions: Adenosine stress CMR, by qualitative and semi-quantitative normalized upslope analyses are reproducible techniques in both patients with multi-vessel CAD and those without known CAD. The robust inter-study reproducibility of perfusion CMR supports its clinical and research application.

\section{Background}

First-pass perfusion cardiovascular magnetic resonance (CMR) allows non-invasive, rapid and ionizing-radiationfree evaluation of myocardial perfusion and, in combination with other CMR techniques, provides a comprehensive assessment of ischemic heart disease. Stress CMR

* Correspondence: s.chih@victorchang.edu.au

1 Victor Chang Cardiac Research Institute, Lower Packer Building, Liverpool Street, Sydney, Australia

Full list of author information is available at the end of the article has high diagnostic accuracy with 91\% sensitivity and $81 \%$ specificity reported for the detection of coronary artery disease (CAD) [1]. Whilst single photon emission computed tomography (SPECT) has been the most widely utilized myocardial perfusion imaging modality, CMR has demonstrated superiority for the detection of CAD [2-4]. In particular, the improved spatial resolution of CMR permits delineation of both small and diffuse areas of subendocardial ischemia in the setting of mild to moderate CAD and multi-vessel disease. In the largest 
multicenter clinical trial to date, the performance of CMR was shown to be comparable to SPECT, and possibly superior in the detection of three-vessel CAD [4]. Furthermore, there is also emerging evidence for CMR as a powerful coronary risk stratification tool. In patients with known or suspected CAD, a normal stress CMR study was shown to predict a $0.8 \%$ risk of cardiac death or nonfatal myocardial infarction (MI) over a three year period, whilst an abnormal result was shown to have a risk proportional to the degree of abnormality [5]. In another study of patients presenting to the emergency department with chest pain and a negative troponin, an abnormal perfusion CMR scan had a 100\% sensitivity and 91\% specificity for predicting CAD or an adverse cardiac event at one year [6].

However, despite the appeal and anticipated future application of perfusion CMR in both clinical and research arenas, its reproducibility remains incompletely evaluated, particularly in the severe CAD patient-population. Reproducibility is a measure of the ability of a test to produce the same result when applied under similar conditions. Thus, reproducibility affects test precision, reliability and is particularly important for tests that are used in serial examinations to evaluate response to therapy or for clinical monitoring. Knowing the inherent variability of a technique is critical for interpreting the significance of changes in measurements. For research applications, tests with poor reproducibility require increased sample sizes to reduce statistical error, which would unfavorably increase study duration and costs.

Thus far, only three small studies have reported the reproducibility of perfusion CMR [7-9]. In a study of 16 subjects ( 7 volunteers and 9 CAD patients), $21 \%$ and $41 \%$ coefficient of variation $(\mathrm{CoV})$ were reported for adenosine stress CMR myocardial perfusion reserve index (MPRi) by Fermi deconvolution and normalized upslope analysis, respectively [7]. Muhling et al. [8] reported good intra- $(R=0.80-0.85)$ and inter-observer $(R=0.83-0.88)$ agreement for good quality images, using the quantitative Fermi deconvolution method. In another study of the qualitative analysis of dobutamine stress CMR in patients with severe Canadian Cardiovascular Society class III-IV angina, an inter-observer agreement, $\mathrm{K}$, of 0.70 , and low intra-subject variability were reported [9]. These small studies combined with high CMR diagnostic performance suggest that perfusion CMR has reasonable reproducibility. Further confirmation is, however, prudent, particularly in clinical research where perfusion CMR is increasingly being employed as a clinical endpoint and accurate estimates of sample size are necessary. Furthermore, in addition to technical factors, reproducibility may also be affected by biological conditions related to the subject, and observer variability associated with subjective assessments. Thus, reproducibility needs to be evaluated in specific patient cohorts and also considered in individual imaging centres due to varying local reporter-expertise, imaging and contrast administration protocols. The purpose of this study was to (1) determine the reproducibility of qualitative and semi-quantitative analyses of first-pass perfusion adenosine stress CMR in patients with multi-vessel CAD versus those at low risk of CAD and; (2) determine sample sizes required for trials utilizing CMR myocardial perfusion as the primary endpoint.

\section{Methods}

The study was conducted between January and September 2009 with the approval of the St Vincent's Hospital Human Research Ethics Committee and with written informed consent from all patients.

\section{Study Design}

Patients underwent two CMR scans that were performed on two separate occasions up to two weeks apart. CMR scans were analyzed in random order by two reporters (JM and SC) who were blinded to patients' details. Qualitative analysis was performed by consensus reporting and semi-quantitative analysis was performed by a single reporter (SC). Inter-study reproducibility was assessed by comparing each patient's first and second scans. Additionally, a single short-axis slice (SA) was chosen from 20 randomly selected scans (10 case and 10 control) and semi-quantitative analysis repeated by the same observer (SC) and by a second observer (JM) to assess intra- and inter-observer reproducibility, respectively.

\section{Patient Population}

Subjects were recruited into one of two groups: CAD (case) or low risk CAD (control). Patients with CAD had angiographically documented multi-vessel CAD ( $\geq 70 \%$ stenosis in $\geq 2$ major coronary vessels ( $>2 \mathrm{~mm}$ diameter)) and Canadian Cardiovascular Society class II-IV angina. Classification of patients in the low risk CAD group was based on them having a Framingham estimated 10 year coronary heart disease risk of $<10 \%$ [10]. Exclusion criteria were alteration in CAD disease management or a new coronary event in the period between the two CMR examinations, contraindication to CMR including incompatible implants or severe claustrophobia, contraindication to adenosine including severe aortic stenosis, conduction disorder (2:1 or greater atrioventricular block) or severe bronchospasm, atrial fibrillation, uncontrolled heart failure, unstable angina or myocardial infarction within 7 days, and significant renal impairment $(\mathrm{GFR} \leq 60 \mathrm{~mL} / \mathrm{min})$.

\section{Cardiovascular Magnetic Resonance Imaging}

CMR was performed at St George Hospital (Kogarah) and Specialist Magnetic Resonance Imaging (Camper- 
down), New South Wales on a 1.5 Tesla scanner (Philips Intera, Best, The Netherlands) using a five channel cardiac phased-array coil, cardiac gating and repeated breath holds. Each examination involved an assessment of resting ventricular function, first-pass perfusion imaging and late gadolinium enhancement (LGE) imaging for infarct delineation.

Cardiac functional imaging was assessed by steadystate free precession cine imaging (balanced turbo field echo) in the long and short axis (SA) planes. The basal SA slice was positioned at the level of the atrioventricular valve in end-diastole, with all subsequent cines acquired in $8 \mathrm{~mm}$ steps towards the apex. Parameters were: echo time $=1.82 \mathrm{~ms}$, repeat time $=3.6 \mathrm{~ms}$, field of view $=350 \times$ $280 \mathrm{~mm}$, read matrix $=192$, phase matrix $=131$, frames $=$ 18 heart phases, flip angle $=70^{\circ}$.

Myocardial perfusion assessment was performed using a T1-weighted single shot gradient echo sequence with a saturation recovery pre-pulse. Parameters were: echo time $=1.06 \mathrm{~ms}$, repetition time $=3 \mathrm{~ms}$, field of view $=360$ $\times 360 \mathrm{~mm}$, read matrix $=128$, phase matrix $=97$, reconstruction matrix $=240$, voxel size $=2.8 \mathrm{~mm} \times 3.6 \mathrm{~mm} \times 8$ $\mathrm{mm}$, flip angle $=20^{\circ}$. Images were acquired over 50 dynamic scans in standard basal, mid and apical SA slices (Figure 1A). LGE imaging for infarct evaluation was performed using an inversion recovery gradient-echo
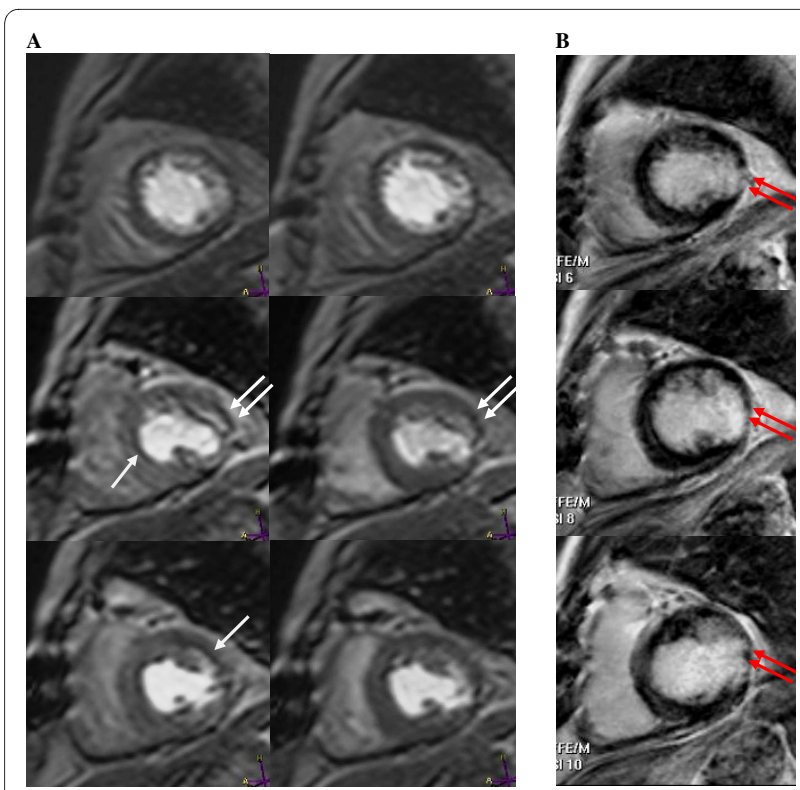

Figure 1 Myocardial first pass perfusion imaging. A, Apical (top row), mid (middle row) and basal (bottom row) short axis slices acquired at stress (left) and rest (right). Inducible ischemia demonstrated by myocardial hypoenhancement, which is visible at stress (single arrow) and absent at rest. Fixed perfusion defects present at stress and rest (white double arrows) corresponding to areas of infarction on $\mathbf{B}$, Late gadolinium enhancement images. Basal to mid lateral infarction (red double arrows) sequence with inversion time optimized to null normal myocardium (Figure 1B).

First-pass perfusion was assessed using three 0.05 $\mathrm{mmol} / \mathrm{kg}$ boluses of gadolinium (Magnevist, Bayer Schering Pharma), administered at peak stress, 10 minutes later at rest (Figure 1A) and immediately after completion of rest imaging (Figure 1B). Gadolinium was delivered via a power injector (Medrad Spectris) at $5 \mathrm{ml} / \mathrm{sec}$ with a $15 \mathrm{ml}$ saline flush. Stress was induced with an intravenous infusion of $140 \mu \mathrm{g} / \mathrm{kg} / \mathrm{min}$ adenosine (Adenoscan, SanofiSynthelabo), commenced up to four minutes prior to stress image acquisition. To ensure maximal vasodilatory responses to adenosine, patients were asked to abstain from caffeinated beverages for 24 hours prior.

\section{Image Analyses}

Image analysis was performed offline on a Philips View Forum workstation using cardiac analysis software. Segmental analysis was based on an 18-segment model: radial division of each of the three $\mathrm{SA}$ slices into six segments with positioning of segment 6 at the insertion of the right ventricle into the inferior septum of the LV (Figure 2A). Image quality was graded as 1 = suboptimal, $2=$ adequate, 3 = good. Measurements for each parameter were reported as a global score (all three SA slice) and according to left anterior descending (LAD), circumflex $(\mathrm{Cx})$ and right coronary artery (RCA) territories as per AHA guidelines.

LV volumes, function and mass were derived by manual tracing of epicardial and endocardial borders of contiguous SA slices at end-diastole and end-systole. The endsystolic phase was visually determined as the one with the smallest cavity area and this was then used for all LV levels. Papillary muscles and trabeculae with visible borders in continuity with the endocardium were included in both mass and volume analyses. Perfusion was assessed qualitatively and semi-quantitatively. An automated image stabilizer was applied to correct for gross cardiac motion and images were windowed appropriately to optimize visualization of perfusion defects, with the same parameters used for both stress and rest images. Qualitative analyses were performed by visual assessment of myocardial signal enhancement in each segment. A perfusion defect was defined as any visible hypoenhancement appearing after maximum signal intensity in the LV blood pool (three frames after the arrival of contrast in the LV cavity) and persisting for at least four dynamics. Perfusion defects were graded by their transmural extent $(1=$ normal, 2 = subendocardial defect, 3 = transmural defect) and degree of hypoenhancement $(1=$ normal (bright), 2 = probably abnormal (grey defect), 3 = definitely abnormal (black defect)). Segments affected by artefact were graded as 0 . An artefact was defined as a defect present at rest but absent at stress, matching stress and rest defects without evidence of infarct on LGE, or 


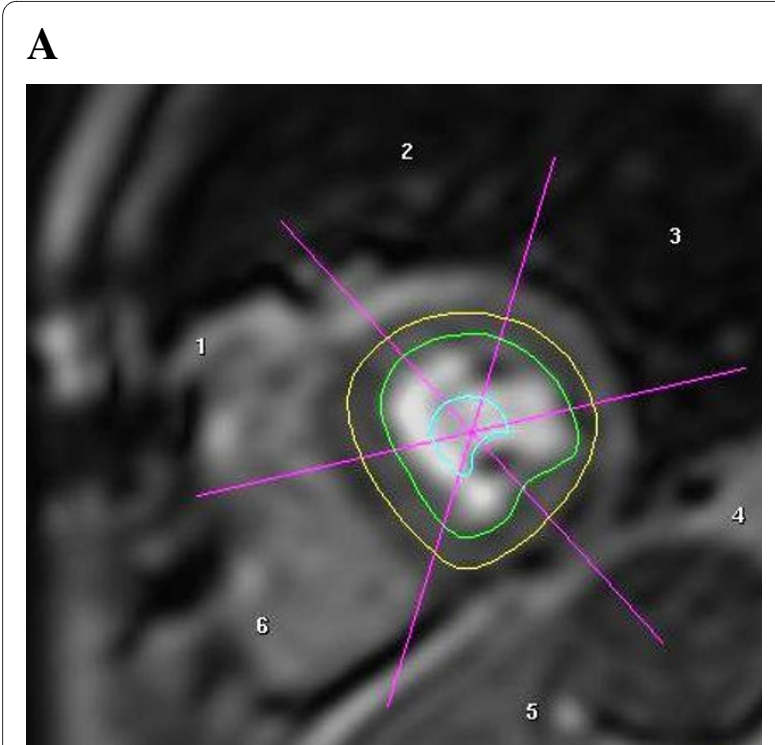

B

Figure 2 Semi-quantitative perfusion analysis. Semi-quantitative perfusion analysis: A, Manual tracing of the endocardial (green) and epicardial (yellow) borders and division of the LV myocardium into 6 segments, and $\mathbf{B}$, Generation of signal intensity (y axis) vs. time ( $x$ axis) curves for each myocardial segment and LV (blue). Maximal upslopes for each myocardial segment, normalized to LV input, with the myocardial perfusion reserve index (MPRi) as the ratio of stress to rest upslopes.

segments in the basal SA slice obtained through the left ventricular outflow tract. Transmural and hypoenhancement ischemia indices, reflecting the degree of reversible ischemia, were calculated as a ratio of summed segmental stress-to-rest scores, thus resulting in a grading scale of 1 = no ischemia, $>1$ and $<3=$ mild ischemia, and $3=$ severe ischemia. Abnormal ischemic segments were defined by segmental transmural ischemia index $>1$. Myocardial perfusion ratio index (MPRi) was derived from maximal upslopes of stress to rest myocardial contrast signal intensity time curves, normalized to LV input (Figure 2B). Myocardial segments in the basal slice obtained through the LV outflow tract were excluded. Segmental infarction was graded as $1=$ normal, $2=$ subendocardial infarct and $3=$ transmural infarct. The infarct index was determined by the summed segmental infarct score divided by the number of segments analyzed.

\section{Statistical Analyses}

Within group differences in adenosine dose, hemodynamic parameters and baseline CMR measures were assessed using paired Student's t-test, and differences in symptomatic response using Fisher's exact test. Reproducibility was defined by the coefficient of variation ( $\mathrm{CoV})$ [11]. This was determined by dividing the standard deviation (SD) of the differences between the two measurements by the average of the two measurements, and was expressed as a percentage. For a continuous variable, inter-study reproducibility between groups was assessed by comparing the squared difference of the two measurements between case and control subjects, with statistical significance being assessed by the Mann-Whitney U test. Where the squared difference of the two measurements equaled zero, half of the next smallest value was entered. For perfusion ordinal data, reproducibility was also assessed by calculating the percentage of reported segments with perfect agreement in ischemia grading (none, mild or severe) between the two studies. Bivariate correlations were calculated using the Spearman correlation coefficient to assess inter-study, inter- and intra-observer agreement for semi-quantitative analyses. Sample sizes were calculated to detect of varying degrees of change in perfusion parameters at an $\alpha$ level of 0.05 and power of 0.9 (http://hedwig.mgh.harvard.edu/sample_size/ size.html). All data were analyzed on SPSS for Windows, release 17.0 (SPSS Inc., Chicago, Illinois).

\section{Results}

\section{Patient Characteristics}

Twenty patients (10 low risk CAD controls and 10 patients with CAD) were recruited. Patient characteristics are shown in Table 1. Patients in the control group were younger, without prior cardiac history and had a mean Framingham risk score of $7 \pm 3 \%$. Patients with CAD had an average of $2.8 \pm 0.4$ native coronary arteries diseased, were symptomatic with CCS class II or III angina and the majority $(n=9,90 \%)$ had suffered a previous myocardial infarct.

\section{Adenosine tolerance and hemodynamic effects}

Adenosine-related side effects were reported by the majority of patients (Table 2). The adenosine infusion 
Table 1: Patient demographics.

\begin{tabular}{|c|c|c|}
\hline Characteristic & Case $(n=10)$ & Control $(n=10)$ \\
\hline Age, years & $68 \pm 13(39-83)$ & $56 \pm 4(52-62)^{*}$ \\
\hline Male & $8(80)$ & $8(80)$ \\
\hline \multicolumn{3}{|l|}{ Vascular Risk Factors } \\
\hline Body mass index, $\mathrm{kg} / \mathrm{m}^{2}$ & $26.6 \pm 6.8(24.0-33.0)$ & $25.9 \pm 3.5(20.4-31.5)$ \\
\hline Diabetes & $4(40)$ & $0(0)$ \\
\hline Hypertension & $4(40)$ & $3(30)$ \\
\hline Dyslipidemia $^{\dagger}$ & $8(80)$ & $1(10)$ \\
\hline Smoking previous/current & $6(60) / 1(10)$ & $4(40) / 0(0)$ \\
\hline Framingham risk score, $\%$ & - & $7 \pm 3(2-10)$ \\
\hline \multicolumn{3}{|l|}{ Cardiac History and Status } \\
\hline CCS class angina class II/III & $4(40) / 6(60)$ & - \\
\hline Myocardial infarction & $9(90)$ & - \\
\hline Number of diseased native vessels & $2.8 \pm 0.4(2-3)$ & - \\
\hline Number of CABG operations & $0.7 \pm 0.5(0-1)$ & - \\
\hline Number of $\mathrm{PCl}$ & $1.3 \pm 1.5(0-5)$ & - \\
\hline Number of cardiac medications daily & $6.9 \pm 1.8(4-8)$ & - \\
\hline
\end{tabular}

Values are number (\%) or mean $\pm \mathrm{SD}$ (range). $\mathrm{CABG}=$ coronary artery bypass graft; $\mathrm{CCS}=$ Canadian Cardiovascular Society; $\mathrm{PCl}=$ percutaneous coronary intervention

${ }^{*} \mathrm{p}<0.05$ for control vs. case.

tPresence of hyperlipidemia or lipid-lowering therapy.

was discontinued if the patient developed severe angina, arrhythmias, hypotension or at the patient's request. Adenosine dosage did not differ significantly between studies in either the case or control group, but was administered for longer in the control compared to case group: $2.20 \pm 0.63$ vs. $1.65 \pm 0.55$ minutes, $\mathrm{p}=0.050$.

\section{Cardiac function and perfusion}

There were no significant differences between control and case patients for baseline LV function, volumes or mass (Table 3). As expected, myocardial perfusion was reduced across all measures in the CAD compared to the control group ( $\mathrm{p}<0.020$ for all measures). In the CAD group, infarction as evidenced by LGE, was detected in $29 \%$ (45 of 156 segments) of all myocardial segments analyzed. In this group, mean global infarct index was $1.35 \pm$ 0.34 and was comparable across all three coronary artery territories: $\mathrm{LAD}=1.22 \pm 0.28, \mathrm{Cx}=1.45 \pm 0.49, \mathrm{RCA}=$ $1.42 \pm 0.54$.

\section{Inter-study reproducibility}

The two CMR studies were performed at a mean of $7.7 \pm$ 2.2 days apart. A total of 40 scans were performed, providing 104 slices (624 segments) for analysis with equal numbers from case and control groups. In four patients, only two slices were analyzable due to a high heart rate preventing acquisition of all three slices $(\mathrm{n}=2)$, breath- holding problems resulting in the apical slice being positioned too distally $(\mathrm{n}=1)$ and LV outflow tract affecting the basal slice $(\mathrm{n}=1)$. In two patients, only a single slice was analyzed due to a combination of high heart rate and LV outflow tract acquisition in one patient, and gating difficulties in another patient. Overall, image quality for perfusion analysis was considered adequate in all patients with a mean grading of $2.7 \pm 0.5$ for the first study and 2.8 \pm 0.4 for the second study. No significant differences in image quality were detected between case and control groups or between the two studies within each group.

\section{$L V$ volume and function}

As shown in Table 4, inter-study reproducibility was consistently high for LV volumes, ejection fraction and mass in both case and control groups. A comparison between control and CAD groups revealed a statistically significant difference in reproducibility only for LV end-diastolic volume, which was slightly lower for CAD compared to control patients: $4.4 \%$ vs. $1.9 \% \mathrm{CoV}$ for case and control group, respectively $(\mathrm{p}=0.018)$.

\section{Qualitative myocardial perfusion analysis}

Reproducibility for all qualitative measures of myocardial perfusion was high, as demonstrated by consistently low $\mathrm{CoVs}$, high percentage of perfect agreement between studies, and small mean difference between studies (Table 5). Reproducibility of perfusion defect assessment 
Table 2: Adenosine tolerance and hemodynamic effects.

\begin{tabular}{|c|c|c|c|c|c|c|}
\hline \multirow[b]{2}{*}{ Parameter } & \multicolumn{3}{|c|}{ Control } & \multicolumn{3}{|c|}{ Case } \\
\hline & Study 1 & Study 2 & $\mathbf{p}$ & Study 1 & Study 2 & $\mathbf{p}$ \\
\hline \multicolumn{7}{|l|}{ Rest } \\
\hline Heart rate, beats/min & $60 \pm 7$ & $67 \pm 10$ & 0.411 & $59 \pm 9$ & $61 \pm 12$ & 0.317 \\
\hline Systolic BP, mmHg & $130 \pm 13$ & $130 \pm 11$ & 0.912 & $138 \pm 25$ & $139 \pm 25$ & 0.874 \\
\hline Diastolic BP, mmHg & $81 \pm 16$ & $82 \pm 12$ & 0.787 & $82 \pm 11$ & $70 \pm 9$ & 0.004 \\
\hline \multicolumn{7}{|l|}{ Peak stress } \\
\hline Heart rate, beats/min & $82 \pm 16$ & $83 \pm 10$ & 0.883 & $76 \pm 19$ & $70 \pm 12$ & 0.063 \\
\hline Systolic BP, mmHg & $131 \pm 12$ & $129 \pm 12$ & 0.537 & $130 \pm 31$ & $135 \pm 20$ & 0.671 \\
\hline Diastolic BP, mmHg & $82 \pm 16$ & $83 \pm 10$ & 0.134 & $71 \pm 14$ & $71 \pm 15$ & 1.000 \\
\hline \multicolumn{7}{|l|}{ Adenosine infusion } \\
\hline Duration, min & $2.3 \pm 0.6$ & $2.2 \pm 0.7$ & 0.583 & $1.7 \pm 0.5$ & $1.7 \pm 0.6$ & 0.204 \\
\hline Chest pain & $4(40)$ & $4(40)$ & 0.133 & $5(50)$ & $4(40)$ & 0.524 \\
\hline Breathlessness & $4(40)$ & $1(10)$ & 0.400 & $5(50)$ & $5(50)$ & 0.206 \\
\hline Flushing, headache, dizziness & $8(80)$ & $8(80)$ & 0.467 & $3(30)$ & $3(30)$ & 0.183 \\
\hline
\end{tabular}

Values are number (\%) or mean $\pm \mathrm{SD}$. BP = blood pressure.

by evaluation of transmural extent (CoV 8.1\%) and degree of hypoenhancement (CoV 9.4\%) were both high; however, the percentage of perfect agreement between studies was higher in the former. Mean difference in number of ischemic myocardial segment between studies was small: $-0.10 \pm 0.57$ for control and $-0.20 \pm 1.14$ for CAD. Reproducibility varied across different coronary artery territories and was generally lower compared to global analysis, and in the CAD compared to control group.

Semi-quantitative myocardial perfusion analysis - MPRi

The inter-study reproducibility for MPRi is shown in Table 6. The between-study correlation for global MPRi was moderate: Spearman's correlation coefficient $r=0.76$ $(\mathrm{p}<0.001)$ for all subjects, $\mathrm{r}=0.69(\mathrm{p}=0.026)$ for control group and $r=0.68$ ( $\mathrm{p}=0.029)$ for case group. MPRi CoV was higher compared to qualitative perfusion parameters. Regional coronary artery analysis was less reproducible compared to global analysis in the CAD group (global $\mathrm{CoV} 23 \%$ vs. regional $19 \%, \mathrm{p}=0.051$ ) but comparable in the control group (global CoV 18\% vs regional CoV 19\%, p $=0.528$ ). There was no difference in MPRi reproducibility between case and control groups ( $\mathrm{p}=0.821)$.

\section{Inter-observer and intra-observer myocardial perfusion} reproducibility

There was good agreement for MPRi between and within observers, with low CoVs and moderate to high correlation coefficients (Table 7). Reproducibility was lower for the CAD versus the control group for both, inter- and intra-observer analysis, with a trend towards statistical significance for inter-observer analysis $(\mathrm{p}=0.056)$.

\section{Sample size estimation}

Within-subject variability estimates for the number of ischemic segments, transmural ischemia index and MPRi are shown by the standard deviation of between-study differences for these parameters (Table 8). As shown, clinical trials utilizing CMR to detect changes in myocardial perfusion would require small sample sizes. For example, it is estimated that a two-group parallel-design trial would require 12 patients (6 vs. 6) to detect a mean difference of two abnormal segments or 74 patients (37 vs. 37) to detect a 0.20 change in MPRi with $90 \%$ power $(2$-alpha $=5 \%)$.

\section{Discussion}

This study demonstrates that adenosine stress perfusion CMR has good reproducibility for both qualitative and semi-quantitative analyses and in both patients with and without CAD. There is limited published data available for the reproducibility of serial myocardial perfusion imaging modalities, especially for CMR. The inter-study reproducibility of CMR has been demonstrated for LV volumes, ejection fraction and mass [11,12], but only three small studies have described the reproducibility of perfusion CMR. Of these, one study evaluated only observer reproducibility [8] and a second study used dobutamine as the pharmacological stress agent [9]. Elkington et al. [7] assessed the inter-study reproducibility for adenosine stress CMR in a cohort of 9 CAD patients and 7 healthy volunteers. They reported a $\mathrm{CoV}$ of $41 \%$ for transmural MPRi and 39\% (inferior segment) to 55\% 
Table 3: Baseline left ventricular cardiac function and perfusion.

\begin{tabular}{|c|c|c|}
\hline Parameter & Control & Case \\
\hline \multicolumn{3}{|l|}{ Left ventricular volumes and function } \\
\hline Ejection Fraction, \% & $69 \pm 8$ & $65 \pm 10$ \\
\hline End diastolic volume, $\mathrm{ml}$ & $146 \pm 17$ & $139 \pm 40$ \\
\hline End systolic volume, $\mathrm{ml}$ & $47 \pm 15$ & $50 \pm 27$ \\
\hline Stroke volume, $\mathrm{ml}$ & $99 \pm 11$ & $88 \pm 21$ \\
\hline Mass, $\mathrm{g}$ & $113 \pm 22$ & $126 \pm 23$ \\
\hline \multicolumn{3}{|l|}{ Myocardial Perfusion - Global } \\
\hline Myocardial perfusion reserve index* & $1.59 \pm 0.58^{\#}$ & $1.13 \pm 0.21$ \\
\hline Number of ischemic segments ${ }^{\dagger}$ & $0.75 \pm 1.53^{\#}$ & $4.60 \pm 2.46$ \\
\hline Transmural ischemia index $\neq$ & $1.10 \pm 0.26^{\#}$ & $1.33 \pm 0.19$ \\
\hline Hypoenhancement ischemia index $\$$ & $1.18 \pm 0.42^{\#}$ & $1.51 \pm 0.23$ \\
\hline Infarct index\| & $1.00 \pm 0.00^{\#}$ & $1.35 \pm 0.34$ \\
\hline
\end{tabular}

Values are mean \pm SD. Measurements are an average of the first and second study.

*Averaged segmental stress:rest maximal normalized upslopes.

tNumber of myocardial segments with reversible ischemia by qualitative analysis.

₹Summed segmental transmural stress:rest score $(1=$ normal, $2=$ subendocardial defect, $3=$ transmural defect).

§Summed segmental hypoenhancement stress:rest score ( $1=$ normal, $2=$ grey defect, $3=$ black defect $)$.

IISummed segmental infarct score divided by number of segments analyzed ( $1=$ normal, $2=$ subendocardial infarct, $3=$ transmural infarct).

$\# p<0.020$ for control vs. case.

(anterior segment) for regional analyses using the normalized upslope method. In contrast, we demonstrated improved reproducibility with an MPRi CoV of $19 \%, 18 \%$, $19 \%$ and $26 \%$ for global, LAD-, Cx- and RCA-territory, respectively. Perfusion findings were generally less reproducible by regional coronary artery analysis compared to global analysis. This may be methodological as global and regional perfusion parameters are derived by summed segmental perfusion measurements divided by the number of segments analyzed (18 for global and 5 to 7 for regional coronary artery territory). Hence, segment variability in measurements between studies will be diluted to a greater extent for global versus regional analyses.

The reproducibility of qualitative analysis was better than that for semi-quantitative analysis by MPRi: global
CoV for all subjects - 19\% for MPRi, $8 \%$ for transmural ischemia index and 9\% for hypoenhancement ischemia index. As fully automated techniques remove observer variability, improved accuracy and uniformity of measures are expected for quantitative compared to qualitative techniques. MPRi by normalized upslope analysis, however, is at best semi-quantitative as it is dependent on the operator to discern endocardial and epicardial borders accurately throughout the cardiac cycle. Furthermore, the occurrence of certain types of artefacts (eg. ectopy, stress and rest slice mismatch) may impede semiquantitative analysis but may be overcome by an experienced reporter on visual inspection.

In comparison to control subjects, patients with known CAD were expected to have lower inter-study reproduc-

Table 4: Inter-study reproducibility for LV volumes and function.

\section{Coefficient of Variation (\%)}

\begin{tabular}{lccc} 
& Control & Case & p* \\
\cline { 2 - 3 } & & 2.7 & 0.292 \\
\hline Left ventricular ejection fraction & 2.4 & 4.4 & 0.018 \\
Left ventricular end diastolic volume & 1.9 & 6.4 & 0.787 \\
Left ventricular end systolic volume & 6.8 & 4.2 & 0.483 \\
Left ventricular stroke volume & 2.1 & 4.0 & 0.619 \\
Left ventricular mass & 4.4 & & \\
\hline
\end{tabular}

${ }^{*}$ Comparison of CoV between control and case. 
Table 5: Inter-study reproducibility for qualitative perfusion parameters.

\begin{tabular}{|c|c|c|c|c|c|c|c|c|c|}
\hline \multirow[b]{2}{*}{ Parameter } & \multicolumn{3}{|c|}{ All Subjects } & \multicolumn{3}{|c|}{ Control } & \multicolumn{3}{|c|}{ Case } \\
\hline & Mean Difference* & CoV & $\begin{array}{l}\text { Perfect } \\
\text { Agreement }^{\dagger}\end{array}$ & Mean Difference* & CoV & $\begin{array}{l}\text { Perfect } \\
\text { Agreement }^{\dagger}\end{array}$ & Mean Difference* & $\mathrm{CoV}$ & $\begin{array}{l}\text { Perfect } \\
\text { Agreement }^{\dagger}\end{array}$ \\
\hline \multicolumn{10}{|c|}{ Number of ischemic segments } \\
\hline Global & $-0.15 \pm 0.88$ & 30.6 & 90.7 & $-0.10 \pm 0.57$ & $75.7^{\ddagger}$ & 96.2 & $-0.20 \pm 1.14$ & 24.7 & 85.3 \\
\hline LAD & $-0.00 \pm 0.65$ & 72.1 & 89.3 & $-0.10 \pm 0.74$ & $210.8^{\ddagger}$ & 95.2 & $-0.10 \pm 0.57$ & 39.2 & 83.3 \\
\hline Circumflex & $-0.05 \pm 0.60$ & 78.0 & 90.3 & $-0.10 \pm 0.32$ & $210.8^{\ddagger}$ & 96.2 & $0.00 \pm 0.82$ & 58.3 & 84.3 \\
\hline $\mathrm{RCA}$ & $-0.07 \pm 0.70$ & 41.0 & 93.0 & $-0.10 \pm 0.32$ & $126.5^{\ddagger}$ & 97.6 & $-0.25 \pm 0.89$ & 27.6 & 88.6 \\
\hline \multicolumn{10}{|c|}{ Transmural ischemia index } \\
\hline Global & $-0.03 \pm 0.10$ & 8.1 & 90.7 & $-0.04 \pm 0.11$ & 9.7 & 96.2 & $-0.03 \pm 0.09$ & 7.1 & 85.3 \\
\hline LAD & $-0.05 \pm 0.29$ & 22.8 & 89.3 & $0.00 \pm 0.15$ & 13.3 & 95.2 & $0.11 \pm 0.38$ & 26.7 & 83.3 \\
\hline Circumflex & $-0.04 \pm 0.21$ & 17.6 & 90.3 & $-0.08 \pm 0.18$ & 16.5 & 96.2 & $0.01 \pm 0.23$ & 18.1 & 84.3 \\
\hline $\mathrm{RCA}$ & $-0.04 \pm 0.17$ & 13.4 & 93.0 & $-0.02 \pm 0.06$ & 5.6 & 97.6 & $-0.07 \pm 0.24$ & 16.8 & 88.6 \\
\hline \multicolumn{10}{|c|}{ Hypoenhancement ischemia index } \\
\hline Global & $-0.04 \pm 0.13$ & 9.4 & 81.1 & $-0.01 \pm 0.06$ & 5.4 & 94.2 & $-0.07 \pm 0.17$ & 11.1 & 67.9 \\
\hline LAD & $0.06 \pm 0.39$ & 28.5 & 78.9 & $0.06 \pm 0.14$ & 12.1 & 90.3 & $-0.04 \pm 0.25$ & 35.1 & 67.2 \\
\hline Circumflex & $-0.05 \pm 0.22$ & 17.3 & 86.4 & $-0.07 \pm 0.21$ & 18.6 & 98.1 & $-0.08 \pm 0.24$ & 17.0 & 74.5 \\
\hline RCA & $-0.11 \pm 0.25$ & 16.8 & 77.9 & $-0.07 \pm 0.14$ & 11.0 & 95.2 & $-0.06 \pm 0.28$ & 19.2 & 61.4 \\
\hline \multicolumn{10}{|l|}{ Infarct index } \\
\hline Global & $-0.00 \pm 0.06$ & 4.7 & 96.0 & $0.00 \pm 0.00$ & 0.0 & 100.0 & $0.00 \pm 0.08$ & 6.1 & 94.2 \\
\hline LAD & $0.06 \pm 0.39$ & 4.2 & 95.4 & $0.00 \pm 0.00$ & 0.0 & 100.0 & $0.00 \pm 0.07$ & 5.5 & 96.7 \\
\hline Circumflex & $-0.05 \pm 0.22$ & 6.4 & 97.1 & $0.00 \pm 0.00$ & 0.0 & 100.0 & $-0.03 \pm 0.11$ & 7.7 & 94.2 \\
\hline $\mathrm{RCA}$ & $-0.11 \pm 0.25$ & 9.6 & 95.3 & $0.00 \pm 0.00$ & 0.0 & 100.0 & $0.04 \pm 0.17$ & 11.8 & 90.9 \\
\hline
\end{tabular}

Values are mean $\pm S D, C o V(\%)$, perfect agreement (\%). LAD = left anterior descending; RCA = right coronary artery.

${ }^{*}$ Mean difference between first and second study.

tPercentage of segments with perfect agreement in ischemia grading between the two studies.

${ }^{\ddagger} \mathrm{CoV}$ is a poor measure of reproducibility due to the average of measurements approximating zero. 
Table 6: Inter-study reproducibility for myocardial perfusion reserve index.

\begin{tabular}{|c|c|c|c|c|c|c|c|}
\hline \multirow[b]{2}{*}{ Parameter } & \multicolumn{2}{|c|}{ All Subjects } & \multicolumn{2}{|l|}{ Control } & \multicolumn{2}{|l|}{ Case } & \multirow[b]{2}{*}{$\mathbf{p}^{\dagger}$} \\
\hline & Mean Difference* & CoV & Mean Difference* & CoV & Mean Difference* & CoV & \\
\hline Global & $0.07 \pm 0.26$ & 18.9 & $-0.01 \pm 0.28$ & 17.6 & $0.15 \pm 0.22$ & 18.8 & 0.850 \\
\hline LAD & $0.07 \pm 0.25$ & 18.4 & $0.03 \pm 0.28$ & 18.1 & $0.12 \pm 0.22$ & 18.9 & 0.384 \\
\hline Circumflex & $0.11 \pm 0.21$ & 19.2 & $0.02 \pm 0.28$ & 17.6 & $0.20 \pm 0.22$ & 19.4 & 0.623 \\
\hline $\mathrm{RCA}$ & $0.01 \pm 0.34$ & 25.6 & $-0.10 \pm 0.32$ & 20.7 & $0.12 \pm 0.33$ & 30.6 & 0.821 \\
\hline
\end{tabular}

Values are mean $\pm \mathrm{SD}$ and $\mathrm{CoV}(\%)$. CoV = coefficient of variation; $\mathrm{LAD}=$ left anterior descending artery; RCA = right coronary artery.

*Mean difference between first and second study.

tComparison of $\mathrm{CoV}$ between control and case.

ibility for a number of reasons, including increased myocardial scarring from prior infarction, previous coronary interventions ( $\mathrm{PCI}$ and $\mathrm{CABG}$ ) generating artefacts, and also poorer clinical condition affecting testing due for example, to an increased susceptibility to ischemia induced arrhythmias or to an inability to breath-hold. As expected, LGE was not detected in the control group but was present in $29 \%$ of myocardial segments analyzed in the CAD group. The mean infarct index was comparable across all three coronary artery territories. Mean differences between studies were higher, and the proportion of myocardial segments with perfect agreement was lower for the CAD compared to the control group. However, despite the presence of moderate scar burden in the CAD compared with control group, there was no significant difference in MPRi reproducibility between the two groups $(p=0.850)$. This suggests that the presence of scar may affect qualitative but not semi-quantitative analysis. Intra-observer reproducibility was similar between the two groups $(\mathrm{p}=0.733)$ and there was only a trend $(\mathrm{p}=$ $0.056)$ towards lower inter-observer reproducibility for MPRi in patients with CAD versus controls: $\mathrm{CoV} 5 \%$ vs. $13 \%$ for control and CAD, respectively. Elkington et al. [7] reported no significant differences in reproducibility between patients with CAD and normal subjects.

The small differences observed between the CAD and control groups in this study may be secondary to a lower than expected reproducibility in the control group. Our control group was selected on the basis of low estimated risk for CAD as defined by a Framingham risk score of $<10 \%$. Therefore, patients in this group may have had undiagnosed underlying CAD. Notably, the mean MPRi was $1.59 \pm 0.58$ for the control group and $1.13 \pm 0.21$ for the CAD group. Previous studies have reported an MPRi of $>2.0$ in normal patients. Al-Saadi et al. [13] reported an $87 \%$ diagnostic accuracy (sensitivity $90 \%$, specificity $83 \%$ ) with a MPRi cutoff value of 1.5 for detecting coronary stenosis $\geq 70 \%$, and Costa et al. [14] showed that a cutoff of 2.04 was $85 \%$ sensitive and $49 \%$ specific in predicting CAD with $\geq 50 \%$ diameter stenosis. We believe that it is important for each centre to define its own MPRi range, given the impact of many variables, such as contrast dose, stress technique and sequence variations on outcome measures.

Small SDs for between-study differences were detected for perfusion parameters, resulting in small sample size estimates for studies using adenosine stress CMR as a primary endpoint. Syed et al. [9] reported for dobutamine CMR in a cohort of CCS III or IV angina patients, a between-study difference of 1.9 myocardial segments and an SD of 1.6, resulting in a sample size of eight being required to detect a change in two abnormal segments. We show similar favorable findings for adenosine CMR with estimated sample sizes of 12 to detect an absolute

Table 7: Inter- and intra-observer reproducibility for MPRi.

\begin{tabular}{|c|c|c|c|c|c|c|c|}
\hline & \multicolumn{2}{|c|}{ All Subjects } & \multicolumn{2}{|c|}{ Control } & \multicolumn{2}{|c|}{ Case } & \multirow[b]{2}{*}{$\mathbf{p}^{*}$} \\
\hline & CoV & $\mathbf{r}$ & CoV & $\mathbf{r}$ & CoV & $\mathbf{r}$ & \\
\hline Inter-observer & 9.0 & $0.93^{\dagger}$ & 5.0 & $0.99^{\dagger}$ & 13.2 & $0.79^{\dagger}$ & 0.056 \\
\hline Intra-observer & 5.3 & $0.94^{\dagger}$ & 4.2 & $0.96^{\dagger}$ & 7.0 & $0.76^{\dagger}$ & 0.733 \\
\hline
\end{tabular}

CoV $(\%)=$ coefficient of variation. $r=$ Spearman's correlation co-efficient.

*Comparison of $\mathrm{CoV}$ between control and case.

${ }^{\dagger} \mathrm{p}<0.02$ 
Table 8: Sample size estimates for myocardial perfusion by CMR.

\begin{tabular}{lcc}
\hline Patient Group & SD* $^{*}$ & Sample Size $^{\dagger}$ \\
\hline All subjects & 0.88 & 12 \\
2 segment change in number of ischemic segments & 0.10 & 46 \\
0.10 change in transmural ischemia index & 0.26 \\
0.20 change in myocardial perfusion reserve index & 0.57 \\
Low risk coronary artery disease - Control & 0.11 & 74 \\
2 segment change in number of ischemic segments & 0.28 \\
0.10 change in transmural ischemia index & 54 \\
0.20 change in myocardial perfusion reserve index & 1.14 \\
Coronary artery disease - Case & 0.09 \\
2 segment change in number of ischemic segments & 0.22 \\
0.10 change in transmural ischemia index & 36 \\
0.20 change in myocardial perfusion reserve index & 54 \\
\hline
\end{tabular}

*Standard deviation of differences between first and second study.

tSample size estimates for a two treatment parallel trial design.

change in two ischemic myocardial segments for all subjects, and of 16 for CAD patients.

Commonly used reproducibility measures have potential shortcomings and require cautious interpretation. We used the $\mathrm{CoV}$ to define reproducibility, a measure that is expressed as a percentage and not in specific units, thereby allowing comparison of variables using different units or scales. The $\mathrm{CoV}$ is calculated from the $\mathrm{SD}$ of the differences in measurements divided by the average. Given that, the denominator is dependent on the average value of measurements, the $\mathrm{CoV}$ loses utility when the average of measurements approximates zero. This limitation was evident when calculating the CoVs for the number of ischemic myocardial segments in the control group in whom this was, as expected, close to zero. The calculated high $\mathrm{CoV}$ of $76 \%$ reflects this drawback and should not be interpreted as poor reproducibility. Inter-study variability for the number of ischemic segments in control subjects is in fact low, as shown by the very small mean difference $(-0.10)$ and SD $(0.57)$, as well as the high percentage of perfect agreements $(96 \%)$ between studies.

\section{Study limitations}

There are several limitations to this study. Currently, CMR does not permit assessment of the entire myocardium. Reproducibility may differ in different myocardial regions and variation in SA slice acquisitions due to variable patient positioning and breath holding can, therefore, affect reproducibility. Furthermore, other groups have performed studies for reproducibility assessment using shorter periods between studies, to minimize physiological variation [11]. Similarly, perfusion CMR was undertaken at two centers and differences in the expertise in these centers may affect data acquisition quality. Reproducibility may have been adversely affected by all of these factors, but nonetheless reflects the practical challenges encountered in both clinical practice and research. Thus, we believe that our assessment of reproducibility by retest at one-to-two week intervals and utilizing two separate imaging centres provides more realistic estimates of the variability to be observed in a longitudinal study. Importantly, our data is limited to a defined perfusion imaging protocol and specific analysis techniques. The use of alternative image acquisition techniques, different contrast agents and dosing, such as dual bolus administration, as well as different techniques for image analysis, such as Fermi deconvolution, may produce significant differences in results for CMR reproducibility. Finally our study was small, based on 10 CAD subjects and 10 controls. This sample size was based on feasibility, and is similar to that used in other typical studies.

\section{Conclusion}

This study demonstrates that adenosine CMR is a reproducible technique with low inter-study and observer variability. Our data provides valuable information for designing adequately powered trials to assess the efficacy of interventions by CMR perfusion and, thus, support the clinical and research applicability of this technique.

\section{Competing interests}

The authors declare that they have no competing interests.

\section{Authors' contributions}

SC and JAM conceived the study. All authors participated in its design. SC and JAM coordinated the study and performed the CMR analysis. SC and ML performed the statistical analysis. SC, JAM, PSM and RMG helped to draft the manuscript. All authors read and approved the final manuscript. 


\section{Acknowledgements}

We thank the magnetic resonance imaging departments at St George Hospital and Specialist Magnetic Resonance Imaging. This study was funded by RT Hall Estate, MBF and St Vincent's Clinic Foundation.

\section{Author Details}

IVictor Chang Cardiac Research Institute, Lower Packer Building, Liverpool Street, Sydney, Australia, 2Department of Cardiology, St Vincent's Hospital, Victoria Street, Sydney, Australia and 'University of New South Wales, Sydney, Australia

Received: 12 April 2010 Accepted: 21 July 2010

Published: 21 July 2010

\section{References}

1. Nandalur KR, Dwamena BA, Choudhri AF, Nandalur MR, Carlos RC: Diagnostic Performance of Stress Cardiac Magnetic Resonance Imaging in the Detection of Coronary Artery Disease: A Meta-Analysis. Journal of the American College of Cardiology 2007, 50:1343-1353.

2. Ishida N, Sakuma H, Motoyasu M, Okinaka T, Isaka N, Nakano T, Takeda K: Noninfarcted myocardium: correlation between dynamic first-pass contrast-enhanced myocardial MR imaging and quantitative coronary angiography. Radiology 2003, 229:209-216.

3. Sakuma H, Suzawa N, Ichikawa Y, Makino K, Hirano T, Kitagawa K, Takeda K: Diagnostic accuracy of stress first-pass contrast-enhanced myocardial perfusion MRI compared with stress myocardial perfusion scintigraphy. AJR Am J Roentgenol 2005, 185:95-102.

4. Schwitter J, Wacker CM, van Rossum AC, Lombardi M, Al-Saadi N, Ahlstrom H, Dill T, Larsson HBW, Flamm SD, Marquardt M, Johansson L: MR-IMPACT: comparison of perfusion-cardiac magnetic resonance with single-photon emission computed tomography for the detection of coronary artery disease in a multicentre, multivendor, randomized trial. Eur Heart J 2008, 29:480-489.

5. Jahnke C, Nagel E, Gebker R, Kokocinski T, Kelle S, Manka R, Fleck E, Paetsch I: Prognostic Value of Cardiac Magnetic Resonance Stress Tests: Adenosine Stress Perfusion and Dobutamine Stress Wall Motion Imaging. Circulation 2007, 115:1769-1776.

6. Ingkanisorn WP, Kwong RY, Bohme NS, Geller NL, Rhoads KL, Dyke CK, Paterson DI, Syed MA, Aletras AH, Arai AE: Prognosis of negative adenosine stress magnetic resonance in patients presenting to an emergency department with chest pain. J Am Coll Cardiol 2006, 47:1427-1432

7. Elkington A, Gatehouse P, Ablitt NA, Yang G, Firmin DN, Pennell DJ: Interstudy reproducibility of quantitative perfusion cardiovascular magnetic resonance. J Cardiovasc Magn reson 2005, 7:815-822.

8. Muhling OM, Dickson ME, Zenovich A, Huang Y, Wilson BV, Wilson RF, Anand IS, Seethamraju RT, Jerosch-Herold M, Wilke NM: Quantitative magnetic resonance first-pass perfusion analysis: inter- and intraobserver agreement. J Cardiovasc Magn Reson 2001, 3:247-256.

9. Syed MA, Paterson DI, Ingkanisorn PW, Rhoads KL: Reproducibility and inter-observer variability of dobutamine stress CMR in patients with severe coronary disease: implications for clinical research. Journal of Cardiovascular Magnetic Resonance 2005, 7:763-768.

10. Wilson PW, D'Agostino RB, Levy D, Belanger AM, Silbershatz H, Kannel WB: Prediction of coronary heart disease using risk factor categories. Circulation 1998, 97:1837-1847.

11. Grothues F, Smith GC, Moon JCC, Bellenger NG, Collins P, Klein HU, Pennell DJ: Comparison of interstudy reproducibility of cardiovascular magnetic resonance with two-dimensional echocardiography in normal subjects and in patients with heart failure or left ventricular hypertrophy. The American Journal of Cardiology 2002, 90:29-34.

12. Bellenger NG, Burgess MI, Ray SG, Lahiri A, Coats AJS, Cleland JGF, Pennell DJ: Comparison of left ventricular ejection fraction and volumes in heart failure by echocardiography, radionuclide ventriculography and cardiovascular magnetic resonance. Are they interchangeable? Eur Heart J 2000, 21:1387-1396.

13. Al-Saadi N, Nagel E, Gross M, Bornstedt A, Schnackenburg B, Klein C, Klimek W, Oswald H, Fleck E: Noninvasive detection of myocardial ischemia from perfusion reserve based on cardiovascular magnetic resonance. Circulation 2000, 101:1379-1383.
14. Costa MA, Shoemaker S, Futamatsu H, Klassen C, Angiolillo DJ, Nguyen M, Siuciak A, Gilmore P, Zenni MM, Guzman L, Bass TA, Wilke N: Quantitative Magnetic Resonance Perfusion Imaging Detects Anatomic and Physiologic Coronary Artery Disease as Measured by Coronary Angiography and Fractional Flow Reserve. J Am Coll Cardiol 2007, 50:514-522.

doi: 10.1186/1532-429X-12-42

Cite this article as: Chih et al., Reproducibility of adenosine stress cardiovascular magnetic resonance in multi-vessel symptomatic coronary artery disease Journal of Cardiovascular Magnetic Resonance 2010, 12:42

\section{Submit your next manuscript to BioMed Central and take full advantage of:}

- Convenient online submission

- Thorough peer review

- No space constraints or color figure charges

- Immediate publication on acceptance

- Inclusion in PubMed, CAS, Scopus and Google Scholar

- Research which is freely available for redistribution
C BioMed Central 